\title{
Enfermedad coronaria en el paciente portador del virus de la inmunodeficiencia humana
}

\author{
René Hameau ${ }^{1}$, Alejandro Aguayo ${ }^{2}$, Pablo Sepúlveda ${ }^{3}$, Osvaldo Pérez ${ }^{1}$, Felipe Sepúlveda ${ }^{4}$, Maurice Cortés ${ }^{3-5}$. \\ 1. Unidad de Medicina Cardiovascular Integrada, Hospital Las Higueras, Talcahuano, Chile. \\ 2. Unidad de Infectología, Servicio de Medicina Interna, Hospital Guillermo Grant Benavente y departamento de Medicina Interna, Universidad de \\ Concepción, Concepción, Chile. \\ 3. División de Enfermedades Cardiovasculares, Centro de Terapia Endovascular, Hospital Clínico Pontificia Universidad Católica, Santiago, Chile. \\ 4. Centro Cardiovascular, Hospital Guillermo Grant Benavente, Concepción, Chile. \\ 5. Unidad de Electrofisiología, Instituto Nacional del Tórax, Santiago, Chile.
}

Las enfermedades cardiovasculares, particularmente la enfermedad coronaria (EC), resultan de especial interés y preocupación en pacientes portadores del virus de la inmunodeficiencia humana (VIH). La ateromatosis acelerada como causa del aumento de prevalencia de enfermedad coronaria ha sido reconocida desde hace varios años en estos pacientes. No obstante, los mecanismos fisiopatológicos involucrados son múltiples y complejos e incluyen factores virales, la respuesta inflamatoria e inmunológica desencadenada por el virus, factores de riesgo cardiovascular tradicionales y efectos de la terapia antirretroviral combinada. Esta revisión de la literatura aborda dichos mecanismos y hace una actualización de los principales estudios clínicos que los sustentan. Se comenta además la evaluación de riesgo cardiovascular y los lineamientos para la revascularización de pacientes con EC portadores de VIH 


\section{Coronary artery disease in patients infected with HIV}

Cardiovascular diseases, particularly coronary artery disease are relevant in patients with the human immunodeficiency virus (HIV). For several years accelerated atheromatosis, a cause of increased prevalence in coronary disease, has been recognized in these patients. However, the pathophysiological mechanisms involved are multiple and complex, including viral factors, the inflammatory and immune response tri- ggered by the virus, traditional cardiovascular risk factors, and the effects of combined antiretroviral therapy. This review of the literature addresses these mechanisms and updates the main clinical studies that support them. Cardiovascular risk assessment and guidelines for revascularization of HIV patients with CHD are also discussed.

Keywords: HIV; Coronary artery disease; Review. 


\section{Introducción:}

La infección por el Virus de la Inmunodeficiencia $\mathrm{Hu}-$ mana (VIH) es una patología que afecta a cerca de 38 millones de personas en el mundo, $90 \%$ de ellas en países en desarrollo. A nivel latinoamericano, es Chile quien lidera las tasas de nuevos contagios en la región, país donde se estima que sólo el $73 \%$ del total de contagiados se encuentran bajo tratamiento ${ }^{1}$.

El riesgo cardiovascular de este grupo es 1,5 a 2 veces superior al de individuos no contagiados y la carga mundial de enfermedades cardiovasculares asociadas a esta patología se han triplicado en las últimas 2 décadas, llegando a constituir hasta el $12 \%$ de la mortalidad global en algunas series. Por otro lado, la enfermedad coronaria en este grupo posee características particulares en cuanto a su fisiopatología y manifestaciones clínicas. El objetivo de este artículo es realizar una revisión actualizada de las bases fisiopatológicas, factores de riesgo y manejo de la patología cardiovascular con énfasis en la enfermedad coronaria asociada a la infección por el virus de la inmunodeficiencia humana.

\section{Fisiopatología de la enfermedad cardiovascular en el paciente portador de VIH.}

Si bien se trata de una enfermedad multifactorial, podemos organizar los factores asociados al desarrollo de la patología cardiovascular en 4 ejes:

\section{1) Envejecimiento de la población.}

Sabemos que la patología coronaria aumenta su prevalencia con la edad y que la terapia antirretroviral actual ha permitido incrementar significativamente la expectativa de vida de estos pacientes. Un informe del Center for Disease Control and Prevention en EEUU, reporta que al año 2018 un 50,7,\% de los pacientes sero-positivos en EEUU eran > 50 años y cerca de 104.457 tenía 65 años o más $(10 \% \text { del total })^{2}$. Por otro lado, la presentación de un Síndrome Coronario Agudo en pacientes portadores del virus suele ser, en promedio, 10 años antes que en la población general lo cual favorece la aparición de eventos recurrentes.

\section{2) Factores de riesgo tradicionales.}

Algunos factores de riesgo cardiovascular son significativamente más prevalentes en los pacientes infectados. A modo de ejemplo, la prevalencia del tabaquismo en este grupo duplica a la población general en Estados Unidos $(42,4 \% \text { vs } 20,6 \%)^{3}$ y los portadores de VIH tienen menor probabilidad de dejar ese hábito. Esta condi- ción también presenta un predominio por el sexo masculino que es un factor asociado a mayor incidencia de enfermedad coronaria.

Tanto la infección como la terapia antirretroviral alteran el perfil lipídico. En primer lugar, el VIH per se ha asociado a un incremento de los Triglicéridos y acidos grasos libres, aun teniendo colesterol total y LDL bajos. También se ha descrito una diferenciación preferencial a macrófagos tipo M1 que pueden favorecer la acumulación de colesterol y disrupción del cap fibroso en la placa aterosclerótica.

Respecto de la Diabetes Mellitus, se trata de una asociación frecuente que puede alcanzar cerca de un 15,1\% en países desarrollados e incluso prevalencias mayores (26-47\%) han sido reportadas en población del África subsahariana ${ }^{4}$. Esta asociación incrementa el riesgo de eventos cardiovasculares hasta en 2,4 veces.

La infección también se ha relacionado a cambios en la composición corporal con aumento de la grasa visceral contribuyendo a la insulino-resistencia y a mayor mortalidad. Se ha descrito un cambio del fenotipo de los pacientes bajo terapia antirretroviral: previamente la terapia se asociaba a una pérdida de tejido adiposo y depósitos ectópicos en hígado - músculo en comparación con la terapia actual que provocaría mayores ganancias de tejido adiposo subcutáneo y visceral (fenotipo VIH-obeso). 5

Los recuentos CD4 < 200 se han asociado a mayor rigidez de la pared aórtica en individuos en etapa SIDA, sin embargo, aun no existe claridad respecto de si la prevalencia de la Hipertensión arterial es superior o no a la población general. En 2017, un metaanálisis ${ }^{6}$ de 63.554 pacientes con VIH mostró que la prevalencia global de Hipertensión fue de 25,2\%; sin embargo, se observaron diferencias según el status terapéutico: $34,7 \%$ en pacientes recibiendo terapia antirretroviral vs $12,7 \%$ en aquellos que no la usaban.

Por último, debemos recalcar que los factores de riesgo tradicionales (hipertensión arterial, diabetes mellitus, tabaquismo, sexo masculino, etc), en especial el antecedente de enfermedad cardiovascular, constituyen los principales factores predictores de infarto agudo al miocardio en este grupo y su impacto es superior al que puede tener el VIH por sí mismo o aquel causado por la terapia antirretroviral moderna.

\section{3) Infección y daño directo por VIH}

La viremia y el grado de disfunción inmune se asocian a mayor riesgo de infarto agudo al miocardio, aún des- 
pués de ajustar por características demográficos y factores de riesgo tradicionales.

Es conocido que los estados de inflamación crónica y activación inmune se asocian a mortalidad y eventos cardiovasculares. El estudio CANTOS $^{7}$ utilizó un inhibidor de Interleukina (IL) 1B (Canakinumab) y mostró que aquellos pacientes que redujeron sus niveles de PCR ultrasensible a valores $<2 \mathrm{mg} / \mathrm{L}$, lograron una reducción del $25 \%$ en eventos cardiovasculares mayores, $31 \%$ en mortalidad cardiovascular y $31 \%$ en mortalidad por cualquier causa.

En el caso de los pacientes viviendo con VIH se ha encontrado que estos presentan niveles aumentados de PCR ultrasensible, Factor de Necrosis tumoral alfa, Interleukina 6 e Interleukina 1B y que estos marcadores de inflamación permanecen elevados, aun con la supresión de la carga viral. Los incrementos en los niveles de Dímero-D y descenso del Fibrinógeno observados en este grupo, también se han asociado a mayor disfunción endotelial en contexto de un estado pro-trombótico. Grund et al. ${ }^{8}$ reportan un aumento en el riesgo del desenlace combinado de muerte y/o condiciones graves no asociadas al virus, de $45 \%$ y $28 \%$ con niveles elevados de Inlerleukina-6 o Dímero-D, respectivamente.

Además, se ha descrito una asociación entre menores recuentos de $\mathrm{CD}^{4}$ con mayor riesgo de Infarto agudo al miocardio y entre una razón $\mathrm{CD} 4 / \mathrm{CD}^{8}$ más bajo con el desarrollo de aterosclerosis coronaria. Sanders et al. ${ }^{9}$ describen también un mayor riesgo de fibrilación / flutter auricular con recuentos bajos de CD4 (OR 1,98; IC95\% 1,21-3,25).

Aún con carga viral suprimida, el riesgo cardiovascular parece ser superior a individuos sin la infección y mayor en aquellos de sexo femenino. A modo de ejemplo, en cohortes del África sub-sahariana, el VIH fue el principal factor de riesgo de accidente cerebrovascular isquémico en población joven ${ }^{10}$. Finalmente, se ha encontrado una asociación entre la coinfección VIH y Hepatis $\mathrm{C}$, con un incremento de eventos cardiovasculares, en particular el riesgo de infartos cerebrales.

\section{4) Terapia antirretroviral.}

La terapia antirretroviral presenta una dualidad particular: Por un lado, se trata de un componente crítico del tratamiento y su descontinuación se ha asociado a incremento en el riesgo de infarto agudo al miocardio; mientras que por otro, el uso de ciertos antirretrovirales, sobre todo los antiguos, se han asociado a mayor riesgo de eventos cardiovasculares.
En el caso de los Inhibidores de la Proteasa (IP) de primera generación se describió un incremento de hasta $10 \%$ en el riesgo de Infarto agudo al miocardio por cada año acumulado de uso (Estudio DAD ${ }^{11}$ ). En particular Indinavir (en absoluto desuso en nuestro país) y Lopinavir/Ritonavir (cada vez menos utilizado) son los IP que han mostrado mayor asociación con eventos cardiovasculares. Por otro lado, Atazanavir no se asoció a mayor riesgo y también redujo la progresión al evaluar el grosor de íntima-media carotídeo al compararlo con Darunavir o Ritonavir a 3 años en pacientes sin Diabetes Mellitus ni patología cardiovascular previa ${ }^{12}$. En la actualidad si se requiere usar un IP, dentro de los disponibles, en pacientes con alto riesgo cardiovascular se debe privilegiar Atazanavir por sobre Darunavir o Lopinavir/Ritonavir.

Respecto de los inhibidores de la transcriptasa reversa análogos de nucleósidos/nucleótidos, existe consenso que los usados actualmente como primera línea, tales como Tenofovir, Lamivudina o Emtricitabina, presentan menos efectos colaterales metabólicos que sus predecesores. En el caso particular del Abacavir, existen publicaciones que asocian su uso a mayor riesgo de un primer infarto agudo al miocardio y guías clínicas lo desaconsejan o al menos, sugieren evitarlo en pacientes de alto riesgo cardiovascular. En otro reporte el uso de Abacavir no se asoció a mayor riesgo de infartos al miocardio recurrentes ${ }^{13}$, por lo que la controversia en este tópico persiste a la fecha.

Del mismo modo, el uso de Efavirenz (Inhibidor de la transcriptasa reversa no nucleósido) también se ha asociado a hipertrigliceridemia, pero Rilpivirina y la recientemente introducida Doravirina se asocian a menos trastornos lipídicos. ${ }^{14}$

Los Inhibidores de la integrasa corresponden a un grupo farmacológico ampliamente usado y recomendado como parte de la terapia antirretroviral de primera línea en diversas guías internacionales. Estos se caracterizan en general por ser potentes y razonablemente seguros, y tanto Raltegravir, Dolutegravir y Bictegravir se asocian a pocos trastornos lipídicos. Sin embargo, Elvitegravir en su coformulación con Cobicistat se asocia a dislipidemia y posee un perfil lipídico comparable a Efavirenz y Atazanavir.

Los potenciales mecanismos que explicarían estos efectos serían la hiperreactividad plaquetaria y disfunción endotelial asociada. A pesar de esto, el impacto del tratamiento a nivel poblacional como causa de eventos cardiovasculares es bajo y pudiera atenuarse con el control 
de los otros factores de riesgo, asociado a una estrategia de prevención primaria que pudiera incluir antiagregantes o hipolipemiantes en individuos de alto riesgo.

\section{Estimación de riesgo cardiovascular}

Existe consenso en que la evaluación del riesgo cardiovascular debe basarse inicialmente en las mismas escalas de riesgo que la población general, por ejemplo, la calculadora de riesgo ASCVD de la AHA/ACC21 o las tablas de Framingham adaptadas a población latinoamericana $^{22}$. En 2014, se crea el puntaje DAD (Data Collection on Adverse Events of anti-HIV drugs) que es específico para evaluar el riesgo cardiovascular en este grupo y que ha sido comparado favorablemente con los puntajes tradicionales, sin bien carece de estudios a largo plazo 23 .

Figura 1: Factores de riesgo y eventos cardiovasculares en pacientes portadores del virus de la inmunodeficiencia humana.

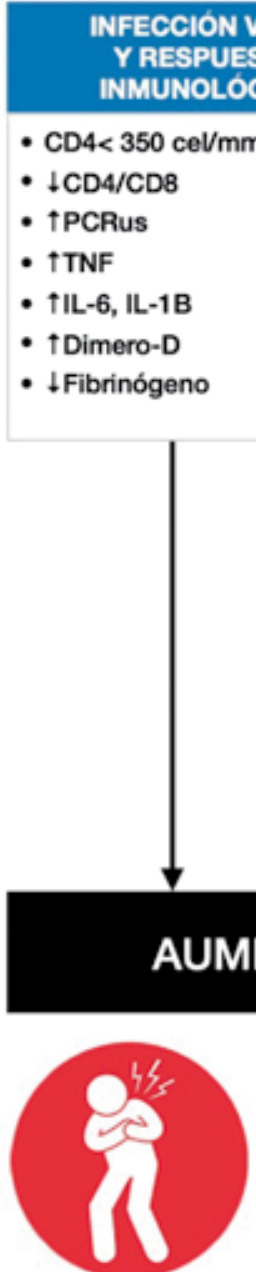

$50 \%$

Infarto Agudo del Miocardio

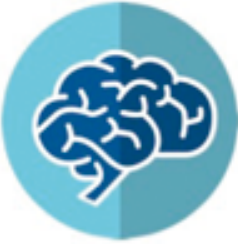

$40 \%$

Accidente Cerebrovascular Isquémico

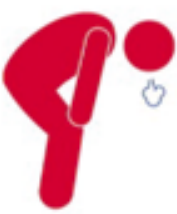

$80 \%$

Insuficiencia Cardiaca

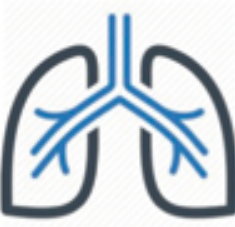

$30 \%$

Hipertensión

Pulmonar
FACTORES DE RIESCO

- FRCV tradicionales

- Aumento expectativa de vida

- Especificos VlH

- Carga vira

- Retraso inicio TARV

- Mala adherencia a tratamiento

- Síndrome metabólico, lipodistrofia/lipoatrofia o esteatosis hepática

- Co-infección por virus Hepatitis C

en usuarios de IP

Simvastatina, Lovastatina

eficacia con IP

- IP disminuyen niveles de Warfarina

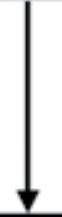

VIH: Virus de la Inmunodeficiencia humana, IP: Inhibidores de la proteasa, PCRus: Proteína C reactiva ultrasensible, IL: Interleukina, TNF: Factor de Necrosis tumoral, FRCV: Factores de riesgo cardiovascular, TARV: Terapia antirretroviral. 
Finalmente, se han descrito factores de riesgo específicos del paciente que vive con VIH:

- Niveles altos de carga viral de forma prolongada y/o retraso en el inicio de la terapia antirretroviral.

- Niveles de CD4 < $350 \mathrm{cel} / \mathrm{mm} 3$.

- Falla de tratamiento o mala adherencia.

- Presencia de Síndrome metabólico, lipodistrofia / lipoatrofia o esteatosis hepática.

- Co-infección con el virus Hepatitis C.

Estos constituirían elementos modificadores que permiten ajustar el riesgo del paciente y son importantes al momento de definir la estrategia terapéutica.

\section{Manifestaciones clínicas}

Todos estos factores se combinan de forma sinérgica y se traducen en que los pacientes portadores del virus presentan un riesgo aumentado de eventos cardiovasculares: (Figura 1)

- Infarto agudo al miocardio (Incremento en casi 50\% del riesgo ${ }^{15}$ ).

- Accidente cerebrovascular isquémico (Incremento hasta $40 \%$ en el riesgo vs población sana).

- Insuficiencia cardíaca (Incremento hasta $80 \%$ en el riesgo ${ }^{16}$ ).

- Hipertensión pulmonar (Incremento de hasta $28 \%$ en el riesgo ${ }^{17}$ ).

- Trombosis venosa (Incremento del riesgo cercano al 39\%).

Tal como se mencionó anteriormente, los pacientes que viven con VIH tienen un riesgo incrementado de Infarto agudo al miocardio y una evolución a largo plazo con mayor recurrencia de eventos cardiovasculares mayores. En base a diversos estudios, se suele describir un perfil "típico" que incluye a un hombre joven (habitualmente $<50$ años), infección conocida de larga data (> 8 años), fumador, dislipidémico $\mathrm{y}$, habitualmente, bajo terapia antirretroviral que incluye un IP. La forma de presentación habitual es como Infarto con supradesnivel del ST (29-64\%), seguido de Infarto sin supradesnivel del ST $(20-48 \%) .^{18}$

Además, se ha descrito que aquellos pacientes que cursan con un síndrome coronario agudo presentan características particulares:

- Menor carga de placa (“plaque burden”) en el árbol coronario $^{19}$.

- Mayor frecuencia de enfermedad coronaria de 1 vaso.

- TIMI risk score más bajo.

- Mayor probabilidad de lesiones coronarias proximales que individuos sin $\mathrm{VIH}^{20}$.

\section{Abordaje Terapéutico}

\section{Farmacología}

Sin olvidar que el pilar del tratamiento lo constituyen las medidas no farmacológicas y la búsqueda de un estilo de vida saludable; es necesario recordar que dada las múltiples alternativas terapéuticas actuales y lo complejo de sus interacciones, debemos tener ciertas consideraciones al momento de elegir los fármacos para el manejo de un paciente coronario con el virus de la inmunodeficiencia humana.

En primer lugar, existe una potencial interacción entre estatinas y los fármacos antirretrovirales. A modo de ejemplo, hay consenso en evitar el uso de Simvastatina y Lovastatina cuya interacción a nivel del citocromo P450 puede incrementar sus niveles hasta 500\%. ${ }^{24}$ Por otro lado, la Rosuvastatina y Atorvastatina tienen poca interacción con la terapia de última generación por lo que su uso no está contraindicado. Finalmente, se esperan los resultados del estudio REPRIEVE ${ }^{25}$ que evaluará el uso de Pitavastatina como estrategia de prevención primaria.

Respecto de la terapia antiagregante, no existe claridad sobre el potencial beneficio de su indicación como prevención primaria; sin embargo, existen algunas interacciones farmacológicas que es necesario mencionar ${ }^{18}$ :

- Ticagrelor se encuentra contraindicado en pacientes usuarios de IP por un riesgo incrementado de hemorragia. - Prasugrel puede ser utilizado bajo vigilancia estrecha. - Clopidogrel presenta interacciones leves con IP (reducción de su eficacia) e interacción con Efavirenz y Etravirina. No existe interacción con otros inhibidores de la transcriptasa reversa no nucleósidos, inhibidores de integrasa o Maraviroc.

Respecto de los fármacos anticoagulantes, se ha observado una disminución de los niveles plasmáticos de Warfarina al interaccionar con IP. Los nuevos anticoagulantes directos se encuentran contraindicados en combinación con IP, excepto Dabigatran.

Finalmente, el manejo de la Diabetes mellitus tipo $2 \mathrm{e}$ Hipertensión arterial debe guiarse por las mismas recomendaciones actuales para la población general. 


\section{Revascularización miocárdica en el paciente por- tador del virus}

Diversas publicaciones han evaluado los resultados de las estrategias de revascularización miocárdica en este grupo. En cuanto a la angioplastía coronaria, la evidencia actual aún no es concluyente respecto de sus resultados en comparación con la población general. D’Ascenzo et al. ${ }^{26}$ publica los resultados de 10.050 pacientes consecutivos infectados y cursando con un Síndrome Coronario Agudo. En este grupo, las tasas de trombosis del stent fueron superiores en aquellos con recuento CD4 $<200 \mathrm{cl} / \mathrm{mm} 3$ (HR 5.9; IC95\% 1,4-2,5; p 0.016) y la ausencia de terapia antirretroviral fue un predictor independiente de muerte cardiovascular (HR 9.9; IC95\% 2.1- 46; p 0.03).

En 2017, un metaanálisis de Bundhun et al ${ }^{27}$ incluyó cerca de 2.268 pacientes (821 sero-positivos vs 1447 sero-negativos) sometidos a angioplastía coronaria. No hubo diferencias significativas en términos de mortalidad global, mortalidad cardíaca, Infarto al miocardio recurrente, accidente cerebrovascular isquémico o eventos cardiovasculares mayores al comparar ambos grupos en un seguimiento hasta 3 años.

Los resultados del uso de stents liberadores de droga son comparables a la población general y su uso en la actualidad es de regla. Ren et al. ${ }^{28}$ evaluaron a 97 pacientes infectados sometidos al implante de DES y los comparó con 97 controles sin la enfermedad. En un seguimiento promedio de 3,1 años, se observaron tasas similares de infarto agudo al miocardio, eventos adversos cardiovasculares mayores o revascularización de la lesión culpable, concluyendo que el beneficio del uso de stents farmacoactivos se mantiene en esta población. Otro estudio en 2017 por Sing et al. ${ }^{29}$ analizó una base de datos norteamericana que incluyó a 7.841 pacientes sero-positivos cursando con un Infarto agudo al miocardio entre los años 2002 a 2013. De ellos, el 71\% era de sexo masculino y un $82 \%$ eran $>50$ años. En los resultados, destaca que estos pacientes tuvieron menos probabilidad de ser sometidos a angioplastía coronaria y menor probabilidad de recibir un stent medicado. Los pacientes con el virus también tuvieron una mayor mortalidad intrahospitalaria ( $12 \%$ vs $10.2 \%$; $<0.001)$, una estadía hospitalaria más prolongada (5 [3-9] días vs 4 [2-7] días; $\mathrm{p}<0,001)$ y costos de hospitalización significativamente más altos (15251,5 dólares vs 12521,7 dólares; $\mathrm{p}<0,001)$.

Respecto de la cirugía de revascularización miocárdica, Boccara et al. ${ }^{30}$ analizaron una base de datos de 5.621 .817 pacientes entre 1998 y 2009 , de los cuales sólo un $0,17 \%$ correspondió a pacientes portadores del VIH intervenidos quirúrgicamente. Estos pacientes presentaron mayor tiempo en circulación extracorpórea (92 min vs 69 min; p 0,01), mayor riesgo de sangrado $(14,8 \%$ vs $2,0 \%$; p 0,03$)$ o de requerir transfusiones sanguíneas (OR 1,19; IC95\% 1,01- 1,40). En este estudio no hubo diferencias en mortalidad ni infarto cerebral, pero los pacientes con el virus tuvieron mayor tasa de eventos adversos cardiovasculares mayores y necesidad de angioplastía coronaria $(35 \%$ vs $11 \%$; p 0,02$)$ en el seguimiento a 41 meses.

A nivel nacional, Muñoz et al. ${ }^{31}$ reporta una serie de 14 pacientes entre 2009 - 2015, sometidos a cirugía cardíaca y que correspondió al $0,54 \%$ del total de pacientes adultos operados en ese período. Los principales diagnósticos fueron: Enfermedad coronaria $(n=8)$ y enfermedad valvular severa $(n=3)$. Cabe destacar que todas las revascularizaciones coronarias se realizaron en el contexto de complicaciones (infarto agudo al miocardio, angina inestable o shock cardiogénico) y que la edad promedio fue de $58+7,2$ años.

\section{Conclusión:}

La infección por el Virus de la inmunodeficiencia humana es una enfermedad crónica que incrementa el riesgo cardiovascular, a pesar de lograr una supresión viral efectiva. Todavía no está totalmente clara la mejor forma de estimar el riesgo cardiovascular en este grupo y se deben tener precauciones al elegir las terapias farmacológicas, especialmente, con estatinas, antiplaquetarios y anticoagulantes, considerando sus interacciones con la terapia antirrretroviral, particularmente con los IP. Los beneficios de la revascularización coronaria parecen ser similares a la población general, debiendo siempre priorizarse el uso de stents liberadores de droga. 


\section{Referencias}

1. MINSAL. Síndrome de la Inmunodeficiencia adquirida VIH/ SIDA. Guía Clínica. 2013. Disponible en: https://www.minsal.cl/sites/default/files/files/GPCVIH.pdf (Consultado el 3 de Enero de 2021).

2. CDC. Diagnoses of HIV Infection in the United States and Dependent Areas 2018. HIV Surveill Rep. 2017;29.

3. MDODO R, FRAZIER E, DUBE S MC, SUTTON M, BROOKS J et al. Cigarette smoking prevalence among adults with HIV compared with the general adult population in the United States: Cross-sectional surveys. Ann Intern Med. 2015;162(5):335-344. doi:10.7326/M14-0954 .

4. NJUGUNA B, KIPLAGAT J, BLOOMFIELD G, PASTAKIA S, VEDANTHAN R KJ. Prevalence, Risk Factors, and Pathophysiology of Dysglycemia among People Living with HIV in Sub-Saharan Africa. J Diabetes Res. 2018;2018(1):1-12.

5. SCHERZER R, HEYMSFIELD S LD, POWDERLY W, TIEN $P$, BACCHETTI $P$ et al. Decreased limb muscle and increased central adiposity are associated with 5-year all-cause mortality in HIV infection. AIDS. 2011;25(11):1405-1414.

6. XU Y, CHEN X WK. Global prevalence of hypertension among people living with HIV: a systematic review and meta-analysis. J Am Soc Hypertens. 2017;11(1):530-540.

7. RIDKER P, EVERETT B, THUREN T, MACFADYEN J, CHANG W, BALLANTYNE C et al. Antiinflammatory therapy with Canakinumab for atherosclerotic disease. N Engl J Med. 2017;377(1):1119-1131.

8. GRUND B, BAKER J, DEEKS S WJ, WENTWORTH D, COZZI-LEPRI A et al. Relevance of interleukin- 6 and D-dimer for serious non-AIDS morbidity and death among HIV-positive adults on suppressive antiretroviral therapy. PLoS One. 2016;11(5):1-7.

9. SANDERS JM, STEVERSON AB, PAWLOWSKI AE, et al.
Atrial arrhythmia prevalence and characteristics for human immunodeficiency virus-infected persons and matched uninfected controls. PLoS One. 2018;13(3):1-12.

10. ABDALLAH A, CHANG J, O'CARROLL C, MUSUBIRE A, CHOW F, WILSON A et al. Stroke in HIV-infected individuals in sub-Saharan Africa (SSA): A systematic review. J Stroke Cerebrovsc Dis. 2018;27(7):1828-1836.

11. FRIIS-M $\varnothing L L E R ~ N$, SABIN C, WEBER R, D'ARMINIO MONFORTE A, EL-SADR W, REISS $P$ et al. Combination Antiretroviral Therapy and the Risk of Myocardial Infarction: The Data Collection on Adverse Events of Anti-HIV Drugs (DAD) Study Group. N Engl J Med. 2003;349(1):1993-2003.

12. STEIN JH, HODIS H, BROWN T, et al. A prospective, randomized clinical trial of antiretroviral therapies on carotid wall thickness: AIDS Clinical Trial Group Study A5260s. J Am Coll Cardiol. 2014;29(14):1775-1783.

13. SABIN C, RYOM L, DÁRMINIO A, HATLEBERG C, PRADIER C, EL-SADR W et al. Abacavir use and risk of recurrent myocardial infarction. AIDS. 2018;32(1):78-88.

14. ADOLESCENTS P ON AG FOR A AND. Guidelines for the Use of Antiretroviral Agents in Adults and Adolescents with HIV.; 2019.

15. FREIBERG M, CHANG C, KULLER L, SKANDERSON M, LOWY E, KRAEMER $\mathrm{K}$ et al. HIV infection and the risk of acute myocardial infarction. JAMA Intern Med. 2013;173(8):614-622.

16. BUTT A, CHANG C, KULLER L, GOETZ M, BIDWELL, LEAF D et al. Risk of heart failure with human immunodeficiency virus in the absence of prior diagnosis of coronary heart disease. Arch Intern Med. 2011;171(8):737-743.

17. BRITTAIN E, DUNCAN M, CHANG J, PATTERSON O, DUVALL S, BRANDT C et al. Increased echocardiographic 
pulmonary pressure in HIV-infected and -uninfected individuals in the veterans aging cohort study. Am J Respir Crit Care Med. 2018;197(7):923-932.

18. BOCCARA F CA. VIH Y CARDIOPATÍA: ¿qué deben saber los cardiólogos? Rev Esp Cardiol. 2016;69(12):1126-1130. doi:10.1016/j.recesp.2016.05.037

19. O'DWYER EJ, BHAMRA-ARIZA P, RAO S, EMMANUEL $S$, CARR A HC. Lower coronary plaque burden in patients with HIV presenting with acute coronary syndrome. Open Hear. 2016;3(2):1-5.

20. THEODOROPOULOS K, MENNUNI M, SARTORI S, MEELU O, YU J, BABER U et al. Quantitative angiographic characterisation of coronary artery disease in patients with human immunodeficiency virus (HIV) infection undergoing percutaneous coronary intervention. EuroIntervention. 2017;12(1):1757-1765.

21. FEINSTEIN M, HSUE P, BENJAMIN L, BLOOMFIELD G, CURRIER J, FREIBERG $M$ et al. Characteristics, Prevention, and Management of Cardiovascular Disease in People Living with HIV: A Scientific Statement from the American Heart Association. Circulation. 2019;140(1):98-124.

22. KUNSTMANN S, LIRA T IG, NÚÑEZ L DGR. Estratificación de riesgo cardiovascular en la población chilena. Rev Médica Clínica Las Condes. 2012;23(6):657-665.

23. MARKOWICZ S, DELFORGE M, NECSOI C DS. Cardiovascular Risk Evaluation of HIV-positive Patients in a Case-Control Study: Comparison of the D:A:D and Framingham Equations. J Int AIDS Soc. 2014;17(4):1-2.

24. MOSEPELE M, MOLEFE-BAIKAI O, GRINSPOON S T V. Benefits and Risks of Statin Therapy in the HIV-Infected Population. Curr Infect Dis Rep. 2018;20(8):1-10.
25. FITCH K, KILEEL E, LOOBY S, ZANNI M, SANCHEZ L, FICHTENBAUM C et al. Successful recruitment of a multi-site international randomized placebo-controlled trial in people with HIV with attention to diversity of race and ethnicity: critical role of central coordination. HIV Res Clin Pract. 2020;21(1):11-23.

26. D'ASCENZO F, CERRATO E AD, MORETTI C, CALCAGNO A, ABOUZAKI N et al., Vetrovec G, et al. Prognostic indicators for recurrent thrombotic events in HIV-infected patients with acute coronary syndromes: use of registry data from 12 sites in Europe, South Africa and the United States. Thromb Res. 2014;134(1):558-564.

27. BUNDHUN P, PURSUN M HW. Does infection with human immunodeficiency virus have any impact on the cardiovascular outcomes following percutaneous coronary intervention?: A systematic review and meta-analysis. BMC Cardiovasc Disord. 2017;17(1):1-7.

28. REN X, TRILESSKAYA M, KWAN DM, NGUYEN K, SHAW RE, HUI PY. Comparison of Outcomes Using Bare Metal Versus Drug-Eluting Stents in Coronary Artery Disease Patients With and Without Human Immunodeficiency Virus Infection. Am J Cardiol. 2009;104(1):216-222.

29. SINGH V, MENDIRICHAGA R SG, RODRIGUEZ A, DABAS N, MUNAGALA A et al. Coronary revascularization for acute myocardial infarction in the HIV population. J Interv Cardiol. 2017;30(5):405-414.

30. BOCCARA F, COHEN A AE, MEULEMAN C, EDERHY S, DUFAITRE G et al. Coronary Artery Bypass Graft in HIV-Infected Patients: A Multicenter Case Control Study. Curr HIV Res. 2008;6(1):59-64.

31. MUÑOZ C, CORSI O, PEREZ C, ZALAQUETT R. Cirugía cardíaca en pacientes infectados por virus de inmunodeficiencia humana. Rev Chil Cardiol 2017;36:17-23 